International Mathematical Forum, Vol. 9, 2014, no. 24, 1149 - 1159

HIKARI Ltd, www.m-hikari.com

http://dx.doi.org/10.12988/imf.2014.4470

\title{
A New View on Soft Normed Spaces
}

\author{
Murat Ibrahim YAZAR \\ Department of Mathematics, Kafkas University, Kars, 36100-Turkey \\ Tunay BILGIN \\ Yuzuncu Yil University, Faculty of Education \\ Department of Secondary Education Science \\ and Mathematics Fields Teaching, Van, Turkey \\ Sadi BAYRAMOV \\ Department of Mathematics, Kafkas University, Kars, 36100-Turkey \\ Cigdem GUNDUZ (ARAS) \\ Department of Mathematics, Kocaeli University, Kocaeli, 41380-Turkey \\ Copyright (c) 2014 Murat Ibrahim YAZAR, Tunay BILGIN, Sadi BAYRAMOV, Çigdem \\ GUNDUZ (ARAS). This is an open access article distributed under the Creative Commons \\ Attribution License, which permits unrestricted use, distribution, and reproduction in any \\ medium, provided the original work is properly cited.
}

\begin{abstract}
In this paper, we work on the structure of soft linear spaces over a field $\mathrm{K}$ and investigate some of its properties. Here, we use the concept of the soft point which was introduced in $[2,6]$. We then introduce the soft norm in soft linear spaces. Finally, we examine the properties of this soft normed space and present some investigations about soft continuous operators in the space.
\end{abstract}

Mathematics Subject Classification: 46B99, 47S99

Keywords: soft norm, soft linear space, soft continuous operators 


\section{Introduction}

Molodtsov [10] introduced the notion of soft set to overcome uncertainties which cannot be dealth with by classical methods in many areas such as environmental science, economics, medical science, engineering and social sciences. This theory is applicable where there is no clearly defined mathematical model. Recently, many papers concerning soft sets have been published; see [1-9].

The concept of soft point was defined in different approaches. Among these, the soft point given in $[2,6]$ is more accurate. Also in the study [6], S.Das and et all introduced the concept of soft metric and investigated some properties of soft metric spaces.

Because of the difficulties to define a vector space over a soft set based upon the concept of soft point S.Das and et all introduced the concept of soft element in [8] and defined a soft vector space by using the concept of soft element. After then they studied on soft normed spaces, soft linear operators, soft inner product spaces and their basic properties [3, 4, 7].

In this paper, by using the concept of soft point we define the soft vector space in a new point of view and investigate some of its properties. We then introduce the soft norm in soft vector spaces. Finally, we examine the properties of the soft normed space and present some investigations about soft continuous linear operators in the space.

\section{Preliminaries}

In this section we recall some basic notations in soft set theory. Let $X$ be an initial universe set and $E$ be a set of all possible parameters under consideration with respect to $X$. The power set of $X$ is denoted by $P(X)$. Molodstov defined the notation of a soft set in the following way:

Definition 1 ([10]) A pair $(F, E)$ is called a soft set over $X$, where $F$ is a mapping given by $F: E \rightarrow P(X)$.

Definition 2 ([9]) A soft set $(F, E)$ over $X$ is said to be a null soft set denoted by $\Phi$, if for all $e \in E, F(e)=\phi$ (null set).

Definition 3 ([9]) A soft set $(F, E)$ over $X$ is said to be an absolute soft set denoted by $\tilde{X}$, if for all $\varepsilon \in E, F(e)=X$.

Definition 4 ([5]) Let $\mathbb{R}$ be the set of real numbers and $B(\mathbb{R})$ be the collection of all non-empty bounded subsets of $\mathbb{R}$ and $E$ taken as a set of parameters. Then a mapping $F: E \rightarrow B(\mathbb{R})$ is called a soft real set. If a soft real set is a singleton soft set, it will be called a soft real number and denoted by $\tilde{r}, \tilde{s}, \tilde{t}$ etc. 
$\tilde{0}, \tilde{1}$ are the soft real numbers where $\tilde{0}(e)=0, \tilde{1}(e)=1$ for all $e \in E$, respectively.

Definition 5 ([5]) Let $\tilde{r}, \tilde{s}$ be two soft real numbers. then the following statements

(i) $\quad \tilde{r} \tilde{\tilde{s}} \tilde{s}$ if $\tilde{r}(e) \leq \tilde{s}(e)$, for all $e \in E$;

(ii) $\quad \tilde{r} \geq \tilde{s}$ if $\tilde{r}(e) \geq \tilde{s}(e)$, for all $e \in E$;

(iii) $\quad \tilde{r} \tilde{<} \widetilde{s}$ if $\tilde{r}(e)<\tilde{s}(e)$, for all $e \in E$;

(iv) $\quad \tilde{r} \tilde{>} \tilde{s}$ if $\tilde{r}(e)>\tilde{s}(e)$, for all $e \in E$;

hold.

Definition 6 ([2, 6]) A soft set $(F, E)$ over $X$ is said to be a soft point if there is exactly one $e \in E$, such that $F(e)=\{x\}$ for some $x \in X$ and $F\left(e^{\prime}\right)=\varnothing$, $\forall e^{\prime} \in E /\{e\}$. It will be denoted by $\tilde{x}_{e}$.

Definition 7 ([2, 6]) Two soft point $\tilde{x}_{e}, \tilde{y}_{e^{\prime}}$ are said to be equal if $e=e^{\prime}$ and $x=y$. Thus $\tilde{x}_{e} \neq \tilde{y}_{e^{\prime}} \Leftrightarrow x \neq y$ or $e \neq e^{\prime}$.

Proposition 1 ([2]) Every soft set can be expressed as a union of all soft points belonging to it. Conversely, any set of soft points can be considered as a soft set.

Let $S P(\tilde{X})$ be the collection of all soft points of $\tilde{X}$ and $\mathbb{R}(E)^{*}$ denote the set of all non-negative soft real numbers.

Definition 8 ([6]) A mapping $\tilde{d}: S P(\tilde{X}) \times S P(\tilde{X}) \rightarrow \mathbb{R}(E)^{*}$ is said to be a soft metric on the soft set $\tilde{X}$ if $\tilde{d}$ satisfies the following conditions:

(M1) $\tilde{d}\left(\tilde{x}_{e_{1}}, \tilde{y}_{e_{2}}\right) \tilde{\geq} \tilde{0}$ for all $\tilde{x}_{e_{1}}, \tilde{y}_{e_{2}} \tilde{\in} \tilde{X}$

(M2) $\tilde{d}\left(\tilde{x}_{e_{1}}, \tilde{y}_{e_{2}}\right)=\tilde{0}$ if and only if $\tilde{x}_{e_{1}}=\tilde{y}_{e_{2}} \tilde{\in} \tilde{X}$

(M3) $\tilde{d}\left(\tilde{x}_{e_{1}}, \tilde{y}_{e_{2}}\right)=\tilde{d}\left(\tilde{y}_{e_{2}}, \tilde{x}_{e_{1}}\right)$ for all $\tilde{x}_{e_{1}}, \tilde{y}_{e_{2}} \tilde{\in} \tilde{X}$

(M4) For all $\tilde{x}_{e_{1}}, \tilde{y}_{e_{2}}, \tilde{z}_{e_{3}} \tilde{\in} \tilde{X}, \tilde{d}\left(\tilde{x}_{e_{1}}, \tilde{z}_{e_{3}}\right) \stackrel{\tilde{\leq}}{\tilde{d}}\left(\tilde{x}_{e_{1}}, \tilde{y}_{e_{2}}\right)+\tilde{d}\left(\tilde{y}_{e_{2}}, \tilde{z}_{e_{3}}\right)$.

The soft set $\tilde{X}$ with a soft metric $\tilde{d}$ is called a soft metric space and denoted by $(\tilde{X}, \tilde{d}, E)$. 


\section{Soft Normed Linear Space}

In this section, by using the concept of soft point we define the soft vector space and soft norm in a new point of view and investigate the properties of the soft normed space.

Let $X$ be a vector space over a field $K(K=\mathbb{R})$ and the parameter set $E$ be the real number set $\mathbb{R}$.

Definition 9 Let $(F, E)$ be a soft set over $X$. The soft set $(F, E)$ is said to be a soft vector and denoted by $\tilde{x}_{e}$ if there is exactly one $e \in E$, such that $F(e)=\{x\}$ for some $x \in X$ and $F\left(e^{\prime}\right)=\varnothing, \forall e^{\prime} \in E /\{e\}$.

The set of all soft vectors over $\tilde{X}$ will be denoted by $S V(\tilde{X})$.

Proposition 2 The set $S V(\tilde{X})$ is a vector space according to the following operations;

1. $\tilde{x}_{e}+\tilde{y}_{e^{\prime}}=(\widetilde{x+y})_{\left(e+e^{\prime}\right)}$ for every $\tilde{x}_{e}, \tilde{y}_{e^{\prime}} \in S V(\tilde{X})$;

2. $\tilde{r} . \tilde{x}_{e}=(\widetilde{r x})_{(r e)}$ for every $\tilde{x}_{e} \in S V(\tilde{X})$ and for every soft real number $\tilde{r}$.

Proof. If $\theta \in X$ is a zero vector and $e=0 \in \mathbb{R}$ then $\tilde{\theta}_{0}$ is a soft zero vector in $S V(\tilde{X})$. Furthermore, $(\widetilde{-x})_{(-e)}$ is the inverse of the soft vector $\tilde{x}_{e}$. It is easy to see that the set $S V(\tilde{X})$ is a vector space.

Definition 10 The set $S V(\tilde{X})$ is called soft vector space.

Definition 11 A set $S=\left\{\tilde{x}_{e_{1}}^{1}, \tilde{x}_{e_{2}}^{2}, \ldots, \tilde{x}_{e_{n}}^{n}\right\}$ of soft vectors in $S V(\tilde{X})$ is said to be linearly independent if the following condition

$$
\tilde{r}_{1} . \tilde{x}_{e_{1}}^{1}+\tilde{r}_{2} . \tilde{x}_{e_{2}}^{2}+\ldots+\tilde{r}_{n} \cdot \tilde{x}_{e_{n}}^{n}=\tilde{\theta}_{0} \Leftrightarrow \tilde{r}_{1}, \tilde{r}_{2}, \ldots, \tilde{r}_{n}=0 .
$$

is satisfied for the soft real numbers $\tilde{r}_{i}, 1 \leq i \leq n$.

Proposition $3 A$ set $S=\left\{\tilde{x}_{e_{1}}^{1}, \tilde{x}_{e_{2}}^{2}, \ldots, \tilde{x}_{e_{n}}^{n}\right\}$ of soft vectors in $S V(\tilde{X})$ is linearly independent if the elements of the set $\left\{x^{1}, x^{2}, \ldots, x^{n}\right\}$ in $X$ are linearly independent and the condition $\left(r_{1} e_{1}+r_{2 .} e_{2}+\ldots+r_{n .} e_{n}\right)=0$ is satisfied.

Proof. For any soft real number $\tilde{r}_{i}, 1 \leq i \leq n$

$$
\begin{aligned}
& \tilde{r}_{1} \tilde{x}_{e_{1}}^{1}+\tilde{r}_{2} \cdot \tilde{x}_{e_{2}}^{2}+\ldots+\tilde{r}_{n} . \tilde{x}_{e_{n}}^{n}=\tilde{\theta}_{0} \\
& \Leftrightarrow \quad\left(r_{1} . x^{1}+r_{2} . \widetilde{x^{2}+\ldots}+r_{n .} x^{n}\right)_{\left(r_{1} . e_{1}+r_{2 .} e_{2}+\ldots+r_{n .} e_{n}\right)}=\tilde{\theta}_{0} \\
& \Leftrightarrow \quad\left(r_{1} \cdot x^{1}+r_{2} . x^{2}+\ldots+r_{n .} x^{n}\right)=\tilde{\theta} \text { and }\left(r_{1 .} e_{1}+r_{2 .} e_{2}+\ldots+r_{n .} e_{n}\right)=0 \\
& \Leftrightarrow \quad \tilde{r}_{1}, \tilde{r}_{2}, \ldots, \tilde{r}_{n}=\tilde{0} .
\end{aligned}
$$


Definition 12 Let $S V(\tilde{X})$ be a soft vector space and $\tilde{M} \subset S V(\tilde{X})$ be a subset. If $\tilde{M}$ is a soft vector space, then $\tilde{M}$ is said to be a soft vector subspace of $S V(\tilde{X})$ and denoted by $S V(\tilde{M}) \tilde{\subset} S V(\tilde{X})$.

Example 1 Let us given a class of soft vectors $\left\{\tilde{x}_{e_{k}}^{k}\right\}_{k=\overline{1, n}}$. Then the space $\left\{\sum_{i=1}^{n} \tilde{r}_{i} \cdot \tilde{x}_{e_{i}}^{i}\right\}$ generated by the class $\left\{\tilde{x}_{e_{k}}^{k}\right\}_{k=\overline{1, n}}$ is a soft vector subspace.

Example 2 If $M \subset X$ is a vector subspace then $S V(\tilde{M}) \subset S V(\tilde{X})$ is a soft vector subspace.

By using the definition of the soft vector, we can give the natural definition of soft norm as follows.

Definition 13 Let $S V(\tilde{X})$ be a soft vector space. Then a mapping

$$
\|\cdot\|: S V(\tilde{X}) \rightarrow \mathbb{R}^{+}(E)
$$

is said to be a soft norm on $S V(\tilde{X})$, if $\|$.$\| satisfies the following conditions:$

(N1). $\left\|\tilde{x}_{e}\right\| \tilde{\geq} \tilde{0}$ for all $\tilde{x}_{e} \tilde{\in} S V(\tilde{X})$ and $\left\|\tilde{x}_{e}\right\|=\tilde{0} \Leftrightarrow \tilde{x}_{e}=\tilde{\theta}_{0}$;

(N2). $\left\|\tilde{r} . \tilde{x}_{e}\right\|=|\tilde{r}|\left\|\tilde{x}_{e}\right\|$ for all $\tilde{x}_{e} \tilde{\in} S V(\tilde{X})$ and for every soft scalar $\tilde{r}$;

(N3). $\left\|\tilde{x}_{e}+\tilde{y}_{e^{\prime}}\right\| \tilde{\leq}\left\|\tilde{x}_{e}\right\|+\left\|\tilde{y}_{e^{\prime}}\right\|$ for all $\tilde{x}_{e}, \tilde{y}_{e^{\prime}} \tilde{\in} S V(\tilde{X})$.

The soft vector space $S V(\tilde{X})$ with a soft norm $\|$.$\| on \tilde{X}$ is said to be a soft normed linear space and is denoted by $(\tilde{X},\|\|$.$) .$

Example 3 Let $X$ be a normed space. In this case, for every $\tilde{x}_{e} \tilde{\in} S V(\tilde{X})$,

$$
\left\|\tilde{x}_{e}\right\|=|e|+\|x\|
$$

is a soft norm.

For every $\tilde{x}_{e}, \tilde{y}_{e^{\prime}} \tilde{\in} S V(\tilde{X})$ and for every soft scalar $\tilde{r}$;

(N1). $\left\|\tilde{x}_{e}\right\|=|e|+\|x\| \tilde{\geq} \tilde{0}$

$$
\left\|\tilde{x}_{e}\right\|=\tilde{0} \Leftrightarrow|e|+\|x\|=\tilde{0} \Leftrightarrow e=0, x=\tilde{\theta} \Leftrightarrow \tilde{x}_{e}=\tilde{\theta}_{0}
$$

$(\mathrm{N} 2) .\left\|\tilde{r} . \tilde{x}_{e}\right\|=\left\|\widetilde{(r . x)_{r e}}\right\|=|r e|+\|r . x\|=|r|(|e|+\|x\|)=|\tilde{r}|\left\|\tilde{x}_{e}\right\|$. 
(N3).

$$
\begin{aligned}
\left\|\tilde{x}_{e}+\tilde{y}_{e^{\prime}}\right\| & =\left\|(\widetilde{x+y})_{\left(e+e^{\prime}\right)}\right\|=\left|e+e^{\prime}\right|+\|x+y\| \\
& \leq|e|+\left|e^{\prime}\right|+\|x\|+\|y\| \\
& =(|e|+\|x\|)+\left(\left|e^{\prime}\right|+\|y\|\right) \\
& =\left\|\tilde{x}_{e}\right\|+\left\|\tilde{y}_{e^{\prime}}\right\| .
\end{aligned}
$$

Definition 14 A sequence of soft vectors $\left\{\tilde{x}_{e_{n}}^{n}\right\}$ in $(\tilde{X},\|\|$.$) is said to be con-$ vergent to $\tilde{x}_{e_{0}}^{0}$, if $\lim _{n \rightarrow \infty}\left\|\tilde{x}_{e_{n}}^{n}-\tilde{x}_{e_{0}}^{0}\right\|=\tilde{0}$ and denoted by $\tilde{x}_{e_{n}}^{n} \rightarrow \tilde{x}_{\lambda_{0}}^{0}$ as $n \rightarrow \infty$.

Definition 15 A sequence of soft vectors $\left\{\tilde{x}_{e_{n}}^{n}\right\}$ in $(\tilde{X},\|\|$.$) is said to be a$ Cauchy sequence if corresponding to every $\tilde{\varepsilon} \tilde{>} \tilde{0}, \exists m \in N$ such that $\left\|\tilde{x}_{e_{i}}^{i}-\tilde{x}_{e_{j}}^{j}\right\| \tilde{<} \tilde{\varepsilon}$, $\forall i, j \geq m$ i.e., $\left\|\tilde{x}_{e_{i}}^{i}-\tilde{x}_{e_{j}}^{j}\right\| \rightarrow \tilde{0}$ as $i, j \rightarrow \infty$.

Proposition 4 Every convergent sequence is a Cauchy sequence.

The proof is straight forward.

Definition 16 Let $(\tilde{X},\|\|$.$) be a soft normed linear space. Then (\tilde{X},\|\|$.$) is$ said to be complete if every Cauchy sequence in $\tilde{X}$ converges to a soft vector of $\tilde{X}$.

Definition 17 Every complete soft normed linear space is called a soft Banach space.

Proposition 5 Every soft normed space is a soft metric space.

Proof. Let $(\tilde{X},\|\|$.$) be a soft normed space. If we define the soft metric by$ $\tilde{d}\left(\tilde{x}_{e}, \tilde{y}_{e^{\prime}}\right)=\left\|\tilde{x}_{e}-\tilde{y}_{e^{\prime}}\right\|$ for every $\tilde{x}_{e}, \tilde{y}_{e^{\prime}} \tilde{\in} S V(\tilde{X})$ then it is clear to show that the soft metric axioms are satisfied.

Theorem 6 Let $\tilde{d}: S V(\tilde{X}) \times S V(\tilde{X}) \rightarrow \mathbb{R}^{+}(E)$ be a soft metric. $S V(\tilde{X})$ is a soft normed space if and only if the following conditions;
a) $\tilde{d}\left(\tilde{x}_{e}+\tilde{z}_{e^{\prime \prime}}, \tilde{y}_{e^{\prime}}+\tilde{z}_{e^{\prime \prime}}\right)=\tilde{d}\left(\tilde{x}_{e}, \tilde{y}_{e^{\prime}}\right)$
b) $\tilde{d}\left(\tilde{r} . \tilde{x}_{e}, \tilde{r} . \tilde{y}_{e^{\prime}}\right)=|\tilde{r}| \tilde{d}\left(\tilde{x}_{e}, \tilde{y}_{e^{\prime}}\right)$ 
satisfied.

Proof. If $\tilde{d}\left(\tilde{x}_{e}, \tilde{y}_{e^{\prime}}\right)=\left\|\tilde{x}_{e}-\tilde{y}_{e^{\prime}}\right\|$, then

$$
\tilde{d}\left(\tilde{x}_{e}+\tilde{z}_{e^{\prime \prime}}, \tilde{y}_{e^{\prime}}+\tilde{z}_{e^{\prime \prime}}\right)=\left\|\tilde{x}_{e}+\tilde{z}_{e^{\prime \prime}}-\tilde{y}_{e^{\prime}}-\tilde{z}_{e^{\prime \prime}}\right\|=\left\|\tilde{x}_{e}-\tilde{y}_{e^{\prime}}\right\|=\tilde{d}\left(\tilde{x}_{e}, \tilde{y}_{e^{\prime}}\right)
$$

and

$$
\tilde{d}\left(\tilde{r} . \tilde{x}_{e}, \tilde{r} . \tilde{y}_{e^{\prime}}\right)=\left\|\tilde{r} \tilde{x}_{e}-\tilde{r} \tilde{y}_{e^{\prime}}\right\|=|\tilde{r}|\left\|\tilde{x}_{e}-\tilde{y}_{e^{\prime}}\right\|=|\tilde{r}| \tilde{d}\left(\tilde{x}_{e}, \tilde{y}_{e^{\prime}}\right)
$$

Suppose that the conditions of the proposition are satisfied . Taking $\left\|\tilde{x}_{e}\right\|=$ $\tilde{d}\left(\tilde{x}_{e}, \tilde{\theta}_{0}\right)$ for every $\tilde{x}_{e} \tilde{\in} S V(\tilde{X})$ we have

(N1). $\left\|\tilde{x}_{e}\right\|=\tilde{d}\left(\tilde{x}_{e}, \tilde{\theta}_{0}\right) \tilde{\geq} \tilde{0}$ and $\left\|\tilde{x}_{e}\right\|=\tilde{d}\left(\tilde{x}_{e}, \tilde{\theta}_{0}\right)=\tilde{0} \Leftrightarrow \tilde{x}_{e}=\tilde{\theta}_{0}$

$(\mathrm{N} 2) .\left\|\tilde{r} \tilde{x}_{e}\right\|=\tilde{d}\left(\tilde{r} \tilde{x}_{e}, \tilde{\theta}_{0}\right)=\tilde{d}\left(\tilde{r} \tilde{x}_{e}, \tilde{r} . \tilde{\theta}_{0}\right)=|\tilde{r}| \tilde{d}\left(\tilde{x}_{e}, \tilde{\theta}_{0}\right)=|\tilde{r}|\left\|\tilde{x}_{e}\right\| ;$

(N3).

$$
\begin{aligned}
\left\|\tilde{x}_{e}+\tilde{y}_{e^{\prime}}\right\| & =\tilde{d}\left(\tilde{x}_{e}+\tilde{y}_{e^{\prime}}, \tilde{\theta}_{0}\right)=\tilde{d}\left(\tilde{x}_{e},-\tilde{y}_{e^{\prime}}\right) \\
& \leq \tilde{d}\left(\tilde{x}_{e}, \tilde{\theta}_{0}\right)+\tilde{d}\left(\tilde{\theta}_{0},-\tilde{y}_{e^{\prime}}\right) \\
& =\left\|\tilde{x}_{e}\right\|+|-\tilde{1}|\left\|\tilde{y}_{e^{\prime}}\right\|=\left\|\tilde{x}_{e}\right\|+\left\|\tilde{y}_{e^{\prime}}\right\| .
\end{aligned}
$$

Definition 18 Let $T: S V(\tilde{X}) \rightarrow S V(\tilde{Y})$ be a soft mapping. Then $T$ is said to be soft linear operator if

(L1). $T$ is additive, i.e., $T\left(\tilde{x}_{e}+\tilde{y}_{e^{\prime}}\right)=T\left(\tilde{x}_{e}\right)+T\left(\tilde{y}_{e^{\prime}}\right)$ for every $\tilde{x}_{e}, \tilde{y}_{e^{\prime}} \tilde{\in} S V(\tilde{X})$,

(L2). $T$ is homogeneous, i.e., for every soft scalar $\tilde{r}, T\left(\tilde{r} \tilde{x}_{e}\right)=\tilde{r} . T\left(\tilde{x}_{e}\right)$ for every $\tilde{x}_{e} \tilde{\in} S V(\tilde{X})$,

Definition 19 The soft operator $T: S V(\tilde{X}) \rightarrow S V(\tilde{Y})$ is said to be soft continuous at $\tilde{x}_{e_{0}}^{0} \tilde{\in} S V(\tilde{X})$ if for every sequence $\left\{\tilde{x}_{e_{n}}^{n}\right\}$ of soft vectors of $\tilde{X}$ with $\tilde{x}_{e_{n}}^{n} \rightarrow \tilde{x}_{e_{0}}^{0}$ as $n \rightarrow \infty$, we have $T\left(\tilde{x}_{e_{n}}^{n}\right) \rightarrow T\left(\tilde{x}_{e_{0}}^{0}\right)$ as $n \rightarrow \infty$. If $T$ is soft continuous at each soft vector of $S V(\tilde{X})$, then $T$ is said to be soft continuous operator.

Definition 20 The soft operator $T: S V(\tilde{X}) \rightarrow S V(\tilde{Y})$ is said to be soft bounded, if there exists a soft real number $\tilde{M}$ such that

$$
\left\|T\left(\tilde{x}_{e}\right)\right\| \tilde{\leq} \tilde{M}\left\|\tilde{x}_{e}\right\|
$$

for all $\tilde{x}_{e} \tilde{\in} S V(\tilde{X})$. 
Theorem 7 The soft operator $T: S V(\tilde{X}) \rightarrow S V(\tilde{Y})$ is soft continuous if and only if it is soft bounded.

Proof. Assume that $T: S V(\tilde{X}) \rightarrow S V(\tilde{Y})$ be soft continuous and $T$ is not soft bounded. Thus, there exists at least one sequence $\left\{\tilde{x}_{e_{n}}^{n}\right\}$ such that

$$
\left\|T\left(\tilde{x}_{e_{n}}^{n}\right)\right\| \tilde{\geq} \tilde{n}\left\|\tilde{x}_{e_{n}}^{n}\right\|
$$

where $\tilde{n}$ is a soft real number. It is clear that $\tilde{x}_{e_{n}}^{n} \neq \tilde{\theta}_{0}$. Let us construct a soft sequence as follows;

$$
\tilde{y}_{e_{n}}^{n}=\frac{\tilde{x}_{e_{n}}^{n}}{\tilde{n}\left\|\tilde{x}_{e_{n}}^{n}\right\|}
$$

It is clear that $\tilde{y}_{e_{n}}^{n} \rightarrow \tilde{\theta}_{0}$ as $n \rightarrow \infty$. Since $T$ is soft continuous, then we have $\left\|T\left(\tilde{y}_{e_{n}}^{n}\right)\right\| \rightarrow \tilde{0}$ as $n \rightarrow \infty$.

$$
\left\|T\left(\tilde{y}_{e_{n}}^{n}\right)\right\|=\left\|T \frac{\tilde{x}_{e_{n}}^{n}}{\tilde{n}\left\|\tilde{x}_{e_{n}}^{n}\right\|}\right\|=\frac{\tilde{1}}{\tilde{n}\left\|\tilde{x}_{e_{n}}^{n}\right\|}\left\|T\left(\tilde{x}_{e_{n}}^{n}\right)\right\| \tilde{>} \frac{\tilde{n}\left\|\tilde{x}_{e_{n}}^{n}\right\|}{\tilde{n}\left\|\tilde{x}_{e_{n}}^{n}\right\|}=\tilde{1}
$$

which is a contradiction.

Conversely, suppose that $T: S V(\tilde{X}) \rightarrow S V(\tilde{Y})$ is soft bounded and the soft sequence $\left\{\tilde{x}_{e_{n}}^{n}\right\}$ is convergent to the $\tilde{x}_{e_{0}}^{0}$. In this case,

$$
\left\|T\left(\tilde{x}_{e_{n}}^{n}\right)-T\left(\tilde{x}_{e_{0}}^{0}\right)\right\|=\left\|T\left(\tilde{x}_{e_{n}}^{n}-\tilde{x}_{e_{0}}^{0}\right)\right\| \tilde{\leq} \tilde{M}\left\|\tilde{x}_{e_{n}}^{n}-\tilde{x}_{e_{0}}^{0}\right\| \rightarrow \tilde{0}
$$

which indicates that $T$ is soft continuous.

Definition 21 Let $T: S V(\tilde{X}) \rightarrow S V(\tilde{Y})$ be a soft continuous operator.

$$
\|T\|=\inf \left\{\tilde{M}:\left\|T\left(\tilde{x}_{e}\right)\right\| \tilde{\leq} \tilde{M}\left\|\tilde{x}_{e}\right\|\right\}
$$

is said to be a norm of $T$.

It is obvious that $\left\|T\left(\tilde{x}_{e}\right)\right\| \tilde{\leq}\|T\|\left\|\tilde{x}_{e}\right\|$.

Theorem 8 Let $T: S V(\tilde{X}) \rightarrow S V(\tilde{Y})$ be a soft operator. Then,

$$
\|T\|=\sup _{\tilde{x}_{e} \neq \tilde{\theta}_{0}} \frac{\left\|T\left(\tilde{x}_{e}\right)\right\|}{\left\|\tilde{x}_{e}\right\|}=\sup _{\left\|\tilde{x}_{e}\right\| \tilde{\leq} 1}\left\|T\left(\tilde{x}_{e}\right)\right\|
$$


Proof. Since $\left\|T\left(\tilde{x}_{e}\right)\right\| \tilde{\leq}\|T\|\left\|\tilde{x}_{e}\right\|$, we have

$$
\sup _{\left\|\tilde{x}_{e}\right\| \tilde{\leq} 1}\left\|T\left(\tilde{x}_{e}\right)\right\| \tilde{\leq} \sup _{\left\|\tilde{x}_{e}\right\| \tilde{\leq} 1}\|T\|\left\|\tilde{x}_{e}\right\| \tilde{\leq}\|T\|
$$

Thus,

$$
\sup _{\left\|\tilde{x}_{e}\right\| \tilde{\leq} 1}\left\|T\left(\tilde{x}_{e}\right)\right\| \tilde{\leq}\|T\|
$$

On the other hand, from the definition of $\|T\|, \forall \tilde{\varepsilon}>\tilde{0}, \exists \tilde{x}_{e}$ such that

$$
\left\|T\left(\tilde{x}_{e}\right)\right\| \tilde{>}(\|T\|-\tilde{\varepsilon})\left\|\tilde{x}_{e}\right\|
$$

let us take $\tilde{y}_{e}=\frac{\tilde{x}_{e}}{\left\|\tilde{x}_{e}\right\|}$, where $\tilde{x}_{e} \neq \tilde{\theta}_{0}$. In this case, we have $\left\|\tilde{y}_{e}\right\|=\tilde{1}$. If we write $\left\|\tilde{x}_{e}\right\| \tilde{y}_{e}$ instead of $\tilde{x}_{e}$ in (3) we have

$$
\begin{gathered}
\left\|T\left(\tilde{y}_{e}\left\|\tilde{x}_{e}\right\|\right)\right\| \tilde{>}(\|T\|-\tilde{\varepsilon})\left\|\tilde{y}_{e}\right\| \tilde{x}_{e}\|\| \Rightarrow \\
\left\|T\left(\tilde{y}_{e}\right)\right\| \tilde{>}(\|T\|-\tilde{\varepsilon})\left\|\tilde{y}_{e}\right\|=(\|T\|-\tilde{\varepsilon}) .
\end{gathered}
$$

If we write $\tilde{x}_{e}$ instead of $\tilde{y}_{e}$ in (4), then

$$
\sup _{\left\|\tilde{x}_{e}\right\| \tilde{\leq} 1}\left\|T\left(\tilde{x}_{e}\right)\right\| \tilde{>}\|T\|-\tilde{\varepsilon} .
$$

Since $\tilde{\varepsilon}$ is arbitary we have

$$
\sup _{\left\|\tilde{x}_{e}\right\| \tilde{\leq} 1}\left\|T\left(\tilde{x}_{e}\right)\right\| \tilde{\geq}\|T\|
$$

From (2) and (5), we have

$$
\|T\|=\sup _{\tilde{x}_{e} \neq \tilde{\theta}_{0}} \frac{\left\|T\left(\tilde{x}_{e}\right)\right\|}{\left\|\tilde{x}_{e}\right\|}=\sup _{\left\|\tilde{x}_{e}\right\| \tilde{\leq} 1}\left\|T\left(\tilde{x}_{e}\right)\right\| .
$$

Theorem 9 Let $T: S V(\tilde{X}) \rightarrow S V(\tilde{Y})$ be a soft operator then $\|T\|$ is a soft norm.

\section{Proof.}

(N1) $\|T\| \tilde{\geq} \tilde{0}$. If $\|T\|=\tilde{0}$, then for all $\tilde{x}_{e} \tilde{\in} S V(\tilde{X})$ we have $T\left(\tilde{x}_{e}\right)=\tilde{\theta}_{0}$ so that $T=\theta$. 
$(\mathrm{N} 2)\|\tilde{r} \cdot T\|=\sup _{\left\|\tilde{x}_{e}\right\|=\tilde{1}}\left\|\tilde{r} \cdot T\left(\tilde{x}_{e}\right)\right\|=\sup _{\left\|\tilde{x}_{e}\right\|=\tilde{1}}|\tilde{r}|\left\|T\left(\tilde{x}_{e}\right)\right\|=|\tilde{r}| \sup _{\left\|\tilde{x}_{e}\right\|=\tilde{1}}\left\|T\left(\tilde{x}_{e}\right)\right\|=$ $\tilde{r} .\|T\|$

$$
\begin{aligned}
\|T+S\| & =\sup _{\left\|\tilde{x}_{e}\right\| \tilde{\underline{1}} \tilde{1}}\left\|(T+S)\left(\tilde{x}_{e}\right)\right\|=\sup _{\| \tilde{x}_{\| \tilde{\leq} \tilde{1}}}\left\|T\left(\tilde{x}_{e}\right)+S\left(\tilde{x}_{e}\right)\right\| \\
& \tilde{\leq} \sup _{\left\|\tilde{x}_{e}\right\| \tilde{\tilde{1}} \tilde{1}}\left\|T\left(\tilde{x}_{e}\right)\right\|+\sup _{\left\|\tilde{x}_{e}\right\| \tilde{\leq} \tilde{1}}\left\|S\left(\tilde{x}_{e}\right)\right\|=\|T\|+\|S\| .
\end{aligned}
$$

Theorem 10 Let $T: S V(\tilde{X}) \rightarrow S V(\tilde{Y})$ and $S: S V(\tilde{Y}) \rightarrow S V(\tilde{Z})$ be two soft operators. Then

a)

$$
\|S \circ T\| \tilde{\leq}\|S\|\|T\|
$$

b) If $T: S V(\tilde{X}) \rightarrow S V(\tilde{X})$ is a soft operator, then

$$
\left\|T^{n}\right\| \tilde{\leq}\|T\|^{n} .
$$

is satisfied.

\section{Proof.}

a)

$$
\begin{aligned}
\|S \circ T\| & =\sup \left\{\left\|(S \circ T)\left(\tilde{x}_{e}\right)\right\|:\left\|\tilde{x}_{e}\right\| \tilde{\leq} \tilde{1}\right\} \\
& =\sup \left\{\left\|S\left(T\left(\tilde{x}_{e}\right)\right)\right\|:\left\|\tilde{x}_{e}\right\| \tilde{\leq} \tilde{1}\right\} \\
& \tilde{\leq} \sup \left\{\|S\| \cdot\left\|T\left(\tilde{x}_{e}\right)\right\|:\left\|\tilde{x}_{e}\right\| \tilde{\leq} \tilde{1}\right\} \\
& \tilde{\leq}\|S\|\|T\|
\end{aligned}
$$

b) If we take $T=S$ then we have $\left\|T^{2}\right\| \tilde{\leq}\|T\|^{2}$. Then $\left\|T^{n}\right\| \tilde{\leq}\|T\|^{n}$ is obtained.

\section{Conclusion}

By using the concept of soft point, the soft vector space defined in a new point of view. Based upon this the soft normed space was given. From the investigated properties of soft continuous linear operators in soft normed space wee see that this properties are parallel to the properties as in classical analysis. 


\section{References}

[1] M.I. Ali, F. Feng, X. Liu, W.K. Min and M. Shabir, On some new operations in soft set theory, Comput. Math. Appl., 49 (2005) 1547 - 1553.

[2] S. Bayramov, C. Gunduz(Aras), Soft locally compact and soft paracompact spaces, Journal of Matheatics and System Science, 3 (2013) 122 130 .

[3] S. Das, P. Majumdar, S.K. Samanta, On soft lineer spaces and soft normed lineer spaces, arXiv:1308.1016 [math.GM].

[4] S. Das and S. K. Samanta, Soft linear operators in soft normed linear spaces, Annals of Fuzzy Mathematics and Informatics, 6(2) (2013) 295 314.

[5] S. Das and S. K. Samanta, Soft real sets, soft real numbers and their properties, J. Fuzzy Math., 20 (3) (2012) 551 - 576.

[6] S. Das and S. K. Samanta, Soft metric, Annals of Fuzzy Mathematics and Informatics, 6(1) (2013) 77 - 94.

[7] S. Das, S. K. Samanta, On soft inner product spaces, Annals of Fuzzy Mathematics and Informatics, 6(1) (2013) 151 - 170.

[8] S. Das and S. K. Samanta, On soft metric spaces, J. Fuzzy Math., accepted.

[9] P.K.Maji, R.Biswas, A.R.Roy, Soft set theory, Comput. Math. Appl., 45 (2003) $555-562$.

[10] D. Molodtsov, Soft set-theory-first results, Comput. Math. Appl., $37(1999) 19-31$.

\section{Received: April 7, 2014}

